\title{
Análisis de la calidad de vida en pacientes con tratamiento renal sustitutivo: influencia de los parámetros analíticos y socioclínicos
}

\author{
Juan Carlos Sánchez González, Marta Barallat García, Sara Torres Paniagua, Bárbara Gaviro Matamoros \\ Fundación Jiménez Díaz. Madrid. España
}

Como citar este artículo:

Sánchez-González JC, Barallat-García M, Torres-Paniagua S, Gaviro-Matamoros B. Análisis de la calidad de vida en pacientes con tratamiento renal sustitutivo: influencia de los parámetros analíticos y socioclínicos. Enferm Nefrol. 2019 Abr-Jun;22(2):159-67

\section{Resumen}

Introducción: La enfermedad renal crónica es una enfermedad en crecimiento y un reto para los países desarrollados ya que produce un importante impacto en la calidad de vida de los pacientes, alterando las actividades cotidianas y provocando cambios en los aspectos físicos, emocionales y sociales.

Objetivos: Evaluar la calidad de vida percibida por los pacientes con enfermedad renal crónica sometidos a tratamiento renal sustitutivo con hemodiálisis o con diálisis peritoneal, y en qué medida son influenciados por los parámetros analíticos y socioclínicos.

Material y Método: Estudio transversal en un grupo de pacientes con enfermedad renal crónica en tratamiento con diálisis peritoneal y hemodiálisis, mediante la cumplimentación del cuestionario de Calidad de Vida en las Enfermedades Renales KDQOL-SF 36. Además se añadieron una serie de variables socioclínicas y parámetros de laboratorio para analizar su posible influencia en la calidad de vida. El análisis estadístico fue realizado con el software estadístico SPSS versión 22.0 para Windows.

Resultados: En nuestra muestra observamos que los dominios de la calidad de vida peor valorados por los pacientes son la salud general, estrés por la enfermedad

Correspondencia:

Juan Carlos Sánchez González

Fundación Jiménez Díaz. Madrid

Avenida Reyes Católicos, 2. 28040 Madrid

E-mail: jcsanchez@fjd.es y rol físico; en cambio, los mejor valorados son el dolor corporal y la salud mental. También se halló mayor repercusión sobre algunos dominios de la calidad de vida si el paciente está en tratamiento con hemodiálisis, si dispone de fístula arteriovenosa como acceso, y cuanto más tiempo lleve en tratamiento renal sustitutivo, e incluso, si están alterados ciertos parámetros analíticos como el Ky/V, urea, potasio o hemoglobina.

Conclusiones: El tratamiento renal sustitutivo con hemodiálisis o diálisis peritoneal genera un impacto enorme en la calidad de vida percibida por el paciente y se debe prestar especial atención a la idoneidad de la técnica elegida en cada momento, a la adecuación del acceso para diálisis, sin descuidar a los pacientes "veteranos" por el hecho de llevar mucho tiempo en tratamiento.

PALABRAS CLAVE: Calidad de vida; hemodiálisis; diálisis peritoneal.

\section{Analysis of quality of life in patients with renal replacement therapy: influence of analytical and socio-clinical parameters}

\section{Abstract}

Background: Chronic kidney disease is a growing disease and a challenge for developed countries, generating an important impact on the patients' quality of life, altering daily activities and causing changes in physical, emotional and social aspects. 
Objectives: To evaluate the quality of life perceived by patients with chronic kidney disease undergoing renal replacement therapy with hemodialysis or peritoneal dialysis, and the influence of analytical and socioclinical parameters.

Material and Method: A cross-sectional study in a group of patients with chronic kidney disease undergoing treatment with peritoneal dialysis and hemodialysis was carried out. Participants completed a Quality of Life Questionnaire on Kidney Disease (KDQOL-SF 36). In addition, several socio-clinical variables and laboratory parameters were studied to analyze their possible influence on quality of life. The statistical analysis was carried out with the statistical package SPSS v.22.0.

Results: In our sample, it was observed that the worst domains of quality of life, valued by the patients, were general health, stress due to illness and physical role; on the other hand, the best valued ones were body pain and mental health. We also found greater repercussion in some domains of quality of life when the patient is in hemodialysis treatment, when the patient has arteriovenous fistula as venous access, and the longer the time of renal replacement therapy; and even if certain analytical parameters such as $\mathrm{Kt} / \mathrm{V}$, urea, potassium or hemoglobin are altered.

Conclusions: Renal replacement therapy using hemodialysis or peritoneal dialysis generates a huge impact on the patient's perceived quality of life. Special attention must be paid to the suitability of the technique chosen for each moment, to the adequacy of the dialysis access, without ignoring the "veteran" patients because they have been in treatment for a long time.

KEYWORDS: quality of life; hemodialysis; peritoneal dialysis.

\section{Introducción}

La enfermedad renal crónica (ERC) es una enfermedad en crecimiento que sigue siendo un reto para los países desarrollados ya que estos enfermos se enfrentan a duros tratamientos que mejoran su salud, aunque no necesariamente mejoran lo que la persona entiende como calidad de vida. Tanto la hemodiálisis (HD) como la diálisis peritoneal (DP) producen un importante impacto en la calidad de vida de los pacientes ya que implican dependencia de un tratamiento sustitutivo para poder vivir, lo que alte$\mathrm{ra}$, en mayor o menor medida, las actividades cotidianas y provoca cambios psicológicos, socio-familiares, económicos y laborales importantes, y un impacto significativo en la calidad de vida de la persona y su familia ${ }^{1-4}$.

Uno de los objetivos del tratamiento de la ERC es la adaptación de los pacientes a los cambios en el estilo de vida y a las limitaciones que esta enfermedad supone, ya que una mejora en la calidad de vida se asocia con efectos positivos tanto a nivel psicológico como a nivel social y emocional en pacientes sometidos a tratamientos de diálisis ${ }^{5-7}$.

Existe gran controversia acerca de la técnica de diálisis que menor impacto genera en la percepción de la calidad de vida de los pacientes, según el tipo de estudio y la muestra seleccionada se obtienen resultados a favor o en contra en ambas técnicas en cuanto a calidad de vida se refiere ${ }^{8-11}$.

De la misma manera, el acceso necesario para realizar la diálisis, ya sea catéter (bien peritoneal o bien de hemodiálisis) o fístula arteriovenosa genera un impacto añadido en la calidad de vida percibida por el paciente, especialmente por los cuidados que requiere y por las complicaciones a las que se está expuesto ${ }^{12}$.

Tanto los parámetros del tratamiento como los valores analíticos de los pacientes influyen directamente en su calidad de vida por arrojar una visión objetiva del estado del paciente. Un ejemplo es la dosis de diálisis que recibe el paciente, cuantificada por el $\mathrm{Kt} / \mathrm{V}$, y que cuanto más alta sea, mejor supuestamente estará dializado y en mejor estado se encontrará el enfermo. Sin embargo, el grupo de colaboración europeo de nefrólogos y geriatras que producen guías de práctica clínica sobre el manejo de pacientes mayores con ERC, defiende que en los ancianos sometidos a diálisis generan menor tasa de toxinas urémicas, reduciendo potencialmente la necesidad de diálisis; y que por lo que se ven más afectados es por la elevada multimorbilidad, concluyendo por tanto que la dosis de diálisis prescrita a esta población debe ser individualizada y no la mínima especificada por las guías clínicas actuales, que podría no ser bien tolerada por ellos y terminar siendo contraproducente ${ }^{10,13}$.

Dada la importancia de que el tratamiento se adapte a la vida del paciente y no al contrario, el objetivo principal de esta investigación fue evaluar la calidad de vida percibida por los pacientes con ERC sometidos a tratamiento renal sustitutivo con hemodiálisis o con diálisis peritoneal. Más específicamente, se pretendió evaluar el impacto en los dominios que caracterizan la calidad de 
vida percibida por los pacientes, que estos tratamientos producen y en qué medida son influenciados por los parámetros analíticos y socioclínicos propuestos.

\section{Material y Método}

Se llevó a cabo un estudio transversal sobre calidad de vida en una muestra de pacientes con ERC en tratamiento de diálisis, utilizando un muestreo por conveniencia.

Se incluyeron los pacientes de la Unidad de Diálisis del Hospital Universitario Fundación Jiménez Díaz (FJD), entre los meses de septiembre y octubre de 2018, que cumplían los criterios de inclusión y que, tras ser informados del estudio a realizar y habiendo otorgado su consentimiento para participar, se comprometieron a colaborar con esta investigación.

Los criterios de inclusión fueron:

- Pacientes diagnosticados de ERC en tratamiento con HD o DP en el servicio de diálisis de la FJD.

- Hombres y mujeres mayores de 18 años.

- Capacidad para comprender los ítems del cuestionario.

- Incluidos al menos 4 meses en programa de tratamiento renal sustitutivo.

Para determinar la calidad de vida se utilizó el cuestionario validado "Kidney Disease Quality of Life (KDQOL SF-36)". Es uno de los instrumentos específicos más utilizados para valorar la calidad de vida en los pacientes con ERC y con tratamiento sustitutivo de la función renal, específicamente en la diálisis peritoneal y la hemodiálisis ${ }^{14-17}$.

Este cuestionario es una versión abreviada del KDQOL, lo cual hace que sea más ameno de contestar y el paciente requiera menos tiempo para cumplimentarlo. Dicha versión fue desarrollada con el propósito de medir la calidad de vida en pacientes con enfermedad renal crónica sometidos a algún programa de diálisis. Consta de 36 ítems divididos en 12 dominios. Los englobados en la parte genérica: función física (ítems 2, 3 y 36), rol físico (ítems 4 y 5), dolor corporal (ítem 8), salud general (ítem 1), vitalidad (ítems 9 y 10), función social (ítem 12), rol emocional (ítems 6 y 7 ) y salud mental (ítems 11 y 34 ); y los englobados en una parte específica: estrés (ítems 13 - 16), sintomatología (ítems 17 - 28), repercusión en la vida diaria (29 - 33) y función sexual (ítem 35 ). El cuestionario se evalúa mediante una escala de tipo Likert, la cual nos permite medir actitudes y conocer el grado de conformidad del paciente con cualquier afirmación que le propongamos. A cada numeración en el cuestionario se le asignó una puntuación desde 0 ("peor" puntuación) hasta 100 ("mejor" puntuación).

Finalmente se sumaron los resultados de los ítems de cada dominio y se calculó la media para saber la puntuación de cada dominio. Cuanto más alta es la puntuación, mejor es la calidad de vida percibida por el paciente ${ }^{18}$.

Para alcanzar el objetivo de nuestro estudio se recogieron las siguientes variables:

- Variables sociodemográficas: edad, sexo, estado civil (casado, viudo, divorciado, soltero), situación laboral (activo, en paro, jubilado, pensionista no jubilado), dificultades económicas (percepción subjetiva del paciente sobre si tiene ingresos económicos bajos; se categorizó en si/no), si vive solo o acompañado en su vivienda, y medio de transporte utilizado para acudir al tratamiento (coche propio, ambulancia, taxi, metro).

- Variables clínicas: etiología de la ERC, tiempo en diálisis (expresado en meses), duración del tratamiento (expresado en horas semanales), técnica de diálisis (HD/DP), tipo de acceso (fístula, catéter, catéter peritoneal), factores de riesgo cardiovascular (hipertensión, diabetes, etc.), trasplante previo (si/ no), números de medicamentos que toma y dosis de eritropoyetina (EPO).

- Parámetros analíticos, obtenidos de la última analítica disponible, con una antigüedad no superior a 1 mes de la fecha de regida de datos: hemoglobina, urea, calcio, fósforo, creatinina, albúmina, sodio, potasio, Kt/V.

El cuestionario propuesto fue cumplimentado por los pacientes mientras realizaban el tratamiento con HD para que no emplearan tiempo extra fuera de su tratamiento. Los pacientes con DP lo cumplimentaron durante las visitas programadas de control y seguimiento.

Parte de la muestra lo cumplimentó sin ayuda, sin embargo, los pacientes que presentaban algún déficit visual o motor recibieron nuestra ayuda para la cumplimentación del mismo mientras se dializaban.

Los parámetros analíticos y socioclínicos fueron recabados de la historia por miembros del equipo investigador. Los pacientes fueron previamente informados sobre el estudio y sus objetivos mediante una hoja informativa, que se les entregó junto al cuestionario, en la cual se señalaba que se asumía el consentimiento a participar de 
aquellos participantes que cumplimentasen el cuestionario. Su participación fue totalmente voluntaria, anónima y en cualquier momento podían revocar su consentimiento a participar en el estudio.

Los datos fueron analizados con el software estadístico IBM SPSS 22.0 para Windows. Para realizar el análisis descriptivo de las variables cualitativas se utilizó la distribución de frecuencias. Las variables cuantitativas que seguían una distribución normal se describieron a través de la media y de la desviación estándar, y aqueIlas que no seguían una distribución normal se expresaron con la mediana y el rango intercuartílico. La prueba de normalidad utilizada fue la de Saphiro Wilk por ser menor de 50 el tamaño muestral.

Para el contraste de hipótesis se empleó la prueba de Mann-Whitney para comparar una variable cuantitativa (dominio) y una cualitativa en dos grupos independientes (técnica de diálisis); la prueba de Kruskal-WaIlis para comparar una variable cuantitativa (dominio) y una cualitativa en más de dos grupos independientes (tipo de acceso para diálisis); se empleó la regresión lineal para estudiar la relación entre una variable cuantitativa dependiente (dominio) y otra variable cuantitativa independiente (parámetro analítico), recogiendo la $p$ en ANOVA, y en el caso de que existiese significación, es decir, que el parámetro clínico influyese en el dominio en concreto, se recogió además la $\mathrm{R}$ cuadrado para explicar el porcentaje de la variabilidad del dominio. Se consideraron significativos aquellos resultados en que el grado de significación resultó inferior al 5\% $(p<0,05)$.

\section{Resultados}

La muestra del estudio estaba formada por 35 pacientes, 24 hombres y 11 mujeres, con una edad media de $66,1 \pm 14,7$ años.

La descripción del resto de variables socioclínicas y parámetros analíticos estudiados en la muestra se recoge en las Tablas 1 y 2.

Según los dominios analizados a partir de las preguntas del cuestionario, se han recogido en la Tabla 3 la media y la desviación estándar (DE) de cada dominio, observando que los dominios peor valorados por los pacientes fueron la salud general, estrés por la enfermedad y rol físico. En cambio, los mejor valorados fueron el dolor corporal y la salud mental.
Tabla 1. Características socioclínicas de la muestra estudiada.

\begin{tabular}{|c|c|}
\hline Estado civil & $\begin{array}{c}60 \% \text { casados; } 17 \% \text { solteros; } 11,5 \% \\
\text { viudos; } 11,5 \% \text { divorciados }\end{array}$ \\
\hline Situación laboral & $\begin{array}{c}48,6 \% \text { jubilados; } 28,6 \% \text { pensionistas } \\
\text { no jubilados; } 11,4 \% \text { en activo; } \\
8,6 \% \text { en paro }\end{array}$ \\
\hline Dificultades económicas & $77 \%$ no dificultades \\
\hline Situación en la vivienda & $\begin{array}{l}77,1 \% \text { viven acompañados; } \\
22,9 \% \text { solos }\end{array}$ \\
\hline Medio de transporte & $\begin{array}{c}31,4 \% \text { coche propio; } 25,7 \% \text { metro; } \\
31,4 \% \text { ambulancia; } 5,7 \% \text { taxi }\end{array}$ \\
\hline Acceso diálisis & $\begin{array}{l}62,8 \% \mathrm{FAV} ; 11,4 \% \text { catéter; } \\
25,7 \% \text { catéter peritoneal }\end{array}$ \\
\hline Técnica de diálisis & $26 \mathrm{HD} ; 9 \mathrm{DP}$ \\
\hline Trasplante riñón previo & $74,3 \%$ no trasplante \\
\hline $\begin{array}{l}\text { Etiología ERC } \\
\text { (ambos grupos) }\end{array}$ & $\begin{array}{c}31,4 \% \text { no filiada; } \\
17,1 \% \text { nefropatía diabética; } \\
2,9 \% \text { hipertensiva; } 8,6 \% \text { poliquistosis; } \\
25,7 \% \text { otras causas }\end{array}$ \\
\hline $\begin{array}{l}\text { Tiempo en diálisis } \\
\text { [mediana }(R Q)]\end{array}$ & 22 (46) meses \\
\hline $\begin{array}{l}\text { Duración del tratamiento } \\
\text { [mediana }(R Q)]\end{array}$ & $12(1,77)$ horas/semana \\
\hline $\begin{array}{l}\text { Número medicamentos } \\
\text { [media }(D E)]\end{array}$ & $8,5(3,4)$ fármacos \\
\hline
\end{tabular}

*RQ: rango intercuartílico. $\mathbf{D E}$ : desviación estándar

Tabla 2. Representación de las puntuaciones medias de los parámetros analíticos.

\begin{tabular}{|lc|}
\hline & Media (DE) \\
\hline Dosis de eritropoyetina (UI) & $2871(3019,3)$ \\
\hline Urea $(\mathrm{mg} / \mathrm{dl})$ & $118,3(29)$ \\
\hline Fósforo $(\mathrm{mg} / \mathrm{dl})$ & $4.9(1,3)$ \\
\hline Albúmina $(\mathrm{g} / \mathrm{dl})$ & $3,8(0,38)$ \\
\hline Potasio $(\mathrm{mmol} / \mathrm{l})$ & $5(0,7)$ \\
\hline Hemoglobina $(\mathrm{g} / \mathrm{l})$ & $11,4(1,3)$ \\
\hline Calcio $(\mathrm{mg} / \mathrm{dl})$ & $8,8(0,8)$ \\
\hline Creatinina $(\mathrm{mg} / \mathrm{dl})$ & $7,2(2,2)$ \\
\hline Sodio $(\mathrm{mmol} / \mathrm{l})$ & $139(3,4)$ \\
\hline Kt/V & $1,64(0,36)$ \\
\hline
\end{tabular}

Asimismo se comprobó la posible asociación entre los dominios que caracterizan la calidad de vida con las variables clínicas más relevantes, observando que la técnica de diálisis, el tipo de acceso y el tiempo en diálisis 
Tabla 3. Representación de las puntuaciones medias de los dominios.

\begin{tabular}{|lc|}
\hline & Media $(\mathrm{DE})$ \\
\hline Función física & $56,9(30,6)$ \\
\hline Rol físico & $48,6(44,5)$ \\
\hline Dolor corporal & $81,4(27,3)$ \\
\hline Salud general & $39,7(20,5)$ \\
\hline Vitalidad & $53,1(30,2)$ \\
\hline Función social & $65,7(30,4)$ \\
\hline Rol emocional & $70,7(38,6)$ \\
\hline Salud mental & $73,4(23,4)$ \\
\hline Estrés por la enfermedad renal & $47,9(26,5)$ \\
\hline Sintomatología & $70,5(16,5)$ \\
\hline Repercusión en la vida diaria & $56,7(24,8)$ \\
\hline Función sexual & $72(39,7)$ \\
\hline
\end{tabular}

*DE: desviación estándar

estuvieron asociados con un cambio en los dominios de la calidad de vida (Tabla 4). En concreto, los pacientes en DP presentaron mayores puntuaciones que los pacientes de HD en algunos dominios de la calidad de vida como la salud general, vitalidad, salud mental, estrés por la enfermedad o repercusión en la vida diaria. De la misma manera, el acceso para diálisis que mayores puntuaciones obtiene en algunos dominios es el catéter peritoneal, por encima del catéter para hemodiálisis y de la FAV (por este orden). Y respecto al tiempo en tratamiento con diálisis, se ha visto que a más tiempo, mayor repercusión en determinados dominios de la calidad de vida como el dolor corporal, salud general y rol emocional.

Por último, se analizó si existía asociación entre los parámetros analíticos y los dominios del cuestionario, encontrando significación en la influencia de parámetros como el $\mathrm{Kt} / \mathrm{V}$, la urea, el potasio o la hemoglobina en algunos aspectos de la calidad de vida (Tabla 5).

\section{Discusión}

A pesar de que nuestra muestra no era muy añosa, se aprecia un gran impacto en la calidad de vida percibida en todos los pacientes en tratamiento con diálisis.

El perfil más habitual en nuestra muestra fue el de hombre, jubilado, que no vive solo y en programa de hemodiálisis. Este mismo perfil se ha descrito en varios estudios, donde se ha demostrado la mayor prevalencia de cuidadoras femeninas, ya sean mujeres o hijas, como concluye la revisión bibliográfica de Arroyo en la que encuentran que la figura del cuidador es femenina en el $89 \%$ de los casos $^{19}$.

Llama la atención que más de la mitad de la muestra acude a su tratamiento por sus medios, probablemente más relacionado con la muestra de los pacientes que están en tratamiento con DP y con la edad media y un bajo-mo-

Tabla 4. Valor $p$ de los contrastes de los dominios con las variables clínicas más relevantes.

\begin{tabular}{|c|c|c|c|c|}
\hline & Técnica diálisis & Acceso diálisis & $\begin{array}{c}\text { Tiempo en diálisis } \\
P(R \text { cuadrado si } p<0,05)\end{array}$ & $\begin{array}{c}\text { Duración diálisis } \\
\mathrm{P}(\mathrm{R} \text { cuadrado si } \mathrm{p}<0,05)\end{array}$ \\
\hline Función física & 0,204 & 0,373 & 0,127 & 0,152 \\
\hline Rol físico & 0,920 & 0,777 & 0,188 & 0,173 \\
\hline Dolor corporal & 0,512 & 0,178 & $0,005(0,218)$ & 0,764 \\
\hline Salud general & 0,021 & 0,042 & $0,000(0,331)$ & 0,204 \\
\hline Vitalidad & 0,026 & 0,010 & 0,192 & 0,225 \\
\hline Función social & 0,249 & 0,367 & 0,175 & 0,113 \\
\hline Rol emocional & 0,833 & 0,977 & $0,014(0,171)$ & 0,282 \\
\hline Salud mental & 0,006 & 0,019 & 0,128 & 0,650 \\
\hline Estrés por la enfermedad renal & 0,016 & 0,051 & 0,078 & 0,735 \\
\hline Sintomatología & 0,427 & 0,559 & 0,077 & 0,574 \\
\hline Repercusión en la vida diaria & 0,001 & 0,004 & 0,059 & 0,245 \\
\hline Función sexual & 0,488 & 0,057 & 0,755 & 0,687 \\
\hline
\end{tabular}


Tabla 5. Asociación de los dominios con los parámetros analíticos por regresión lineal.

\begin{tabular}{|c|c|c|c|c|c|c|c|c|c|c|}
\hline & Dosis EPO & Urea & Fósforo & Albúmina & $\mathbf{K}$ & $\mathrm{Hb}$ & $\mathrm{Ca}$ & Creatinina & $\mathrm{Na}$ & $\mathrm{Kt} / \mathrm{V}$ \\
\hline \multicolumn{11}{|c|}{$P(R$ cuadrado si $p<0,05)$} \\
\hline Función física & 0,790 & 0,429 & 0,361 & 0,527 & $\begin{array}{c}0,049 \\
(0,136)\end{array}$ & 0,623 & 0,903 & 0,735 & 0,528 & 0,070 \\
\hline Rol físico & 0,342 & 0,082 & 0,912 & 0,744 & 0,141 & 0,856 & 0,615 & 0,965 & 0,638 & $\begin{array}{c}0,023 \\
(0,232)\end{array}$ \\
\hline Dolor corporal & 0,950 & 0,407 & 0,960 & 0,401 & $\begin{array}{c}0,008 \\
(0,232)\end{array}$ & 0,131 & 0,770 & 0,833 & 0,394 & $\begin{array}{c}0,036 \\
(0,201)\end{array}$ \\
\hline Salud general & 0,517 & 0,947 & 0,665 & 0,312 & 0,122 & 0,209 & 0,508 & 0,398 & 0,073 & $\begin{array}{c}0,018 \\
(0,262)\end{array}$ \\
\hline Vitalidad & 0,702 & 0,652 & 0,333 & 0,308 & 0,300 & 0,462 & $\begin{array}{c}0,027 \\
(0,169)\end{array}$ & 0,676 & 0,126 & 0,075 \\
\hline Función social & 0,121 & $\begin{array}{c}0,045 \\
(0,136)\end{array}$ & 0,100 & 0,932 & 0,121 & $\begin{array}{c}0,043 \\
(0,143)\end{array}$ & 0,439 & 0,205 & 0,242 & 0,134 \\
\hline Rol emocional & 0,516 & 0,124 & 0,339 & 0,863 & 0,318 & 0,385 & 0,413 & 0,832 & 0,702 & 0,667 \\
\hline Salud mental & 0,951 & 0,665 & 0,162 & 0,116 & 0,050 & 0,121 & 0,619 & 0,807 & 0,062 & 0,762 \\
\hline Estrés por la ERC & 0,876 & 0,985 & 0,612 & 0,596 & 0,069 & $\begin{array}{c}0,018 \\
(0,191)\end{array}$ & 0,251 & 0,296 & 0,091 & 0,459 \\
\hline Sintomatología & 0,611 & 0,688 & 0,438 & 0,241 & 0,055 & 0,128 & 0,509 & 0,676 & 0,155 & 0,173 \\
\hline $\begin{array}{l}\text { Repercusión } \\
\text { vida diaria }\end{array}$ & 0,547 & 0,074 & 0,732 & 0,262 & 0,165 & 0,385 & 0,765 & 0,439 & 0,142 & 0,130 \\
\hline Función sexual & 0,763 & $\begin{array}{c}0,014 \\
(0,266)\end{array}$ & $\begin{array}{c}0,020 \\
(0,279)\end{array}$ & 0,529 & 0,308 & 0,781 & 0,523 & 0,505 & 0,722 & 0,341 \\
\hline
\end{tabular}

*K: potasio; Hb: hemoglobina; Ca: calcio; Na: sodio

derado grado de dependencia de la muestra que seguro permite a los pacientes en mejores condiciones acudir al tratamiento por sus medios y así evitar los excesos de tiempo en los desplazamientos y esperas atribuidas al transporte sanitario.

Respecto a los dominios con los que se valora la calidad de vida percibida por los pacientes de nuestra muestra, resultó llamativo que el dominio que menos afecta a su calidad de vida es el dolor corporal, probablemente por ser controlado con analgésicos y por dar más importancia a otros dominios como es la salud vista desde un punto de vista general, aspecto que ve el paciente como lo más afectado por el tratamiento con diálisis. Otros dominios relacionados con la calidad de vida que el paciente percibe como muy mermados son el estrés producido por la enfermedad, y el rol físico.

Realmente en los estudios realizados sobre calidad de vida, se acaban afectando todos los dominios en función de la muestra estudiada en cada estudio ${ }^{20-23}$.

Debido a que el volumen de nuestra muestra, en cuanto a técnica de diálisis se refiere, no era comparable por ser la muestra de HD casi tres veces superior que la de DP, no se pudieron establecer diferencias concluyentes entre ambas técnicas en términos de calidad de vida. Sin embargo, si comparásemos ambas muestras respecto a la técnica de diálisis, da la sensación de que la DP se erige como la modalidad de tratamiento que menor impacto genera en la calidad de vida percibida por los pacientes. Según los diferentes ítems evaluados en nuestro cuestionario, se pudo considerar que la salud general de los participantes en DP era mejor que los sometidos a tratamiento en HD. A nivel emocional, tuvieron menos problemas en su día a día y para realizar las actividades de la vida diaria, probablemente asociado a que el nivel de estrés por la enfermedad renal de estos participantes es menor según el apartado evaluado, y por tanto, también obtuvieron una mayor vitalidad. La comparación de técnicas dialíticas ha sido el objetivo principal de muchas investigaciones, obteniendo resultados dispares en función de la muestra estudiada24-28.

Otra variable descrita en la literatura como influyente en la calidad de vida es el tipo de acceso de diálisis. La mayor parte de la muestra tiene fístula arteriovenosa como acceso para hemodiálisis, variable que puede ayu- 
dar a que el impacto del tratamiento sobre los dominios de la calidad de vida sea menor por haberse demostrado que la fístula arteriovenosa nativa es el acceso vascular de elección entre los pacientes en HD, según la Guía Clínica Española del Acceso Vascular para Hemodiálisis ${ }^{12}$. A pesar de ello, si analizásemos en nuestra muestra de manera aislada la variable "acceso para diálisis", el catéter peritoneal resultaría el acceso que menor impacto causa en determinados dominios de la calidad de vida, aunque teniendo en cuanta todas las variables estudiadas, probablemente sea la técnica de DP en sí misma la que determine una menor afectación de la calidad de vida.

En nuestra muestra, aunque la duración de la diálisis de los pacientes de HD es menor según la mediana de horas analizadas ( 11,66 horas semanales frente a las 40,25 del grupo de peritoneal), debemos tener en cuenta que estos pacientes tienen que acudir varios días a la semana al centro de diálisis, dedicando parte de su tiempo durante el traslado al centro, además de las esperas hasta comenzar la sesión, y el tiempo de espera a la ambulancia en el caso de que sea necesaria, pudiendo ser más difícil compatibilizarlo con sus actividades de la vida diaria y sociales. Sin embargo, las sesiones de DP, según los pacientes del estudio, se pueden adaptar más a la vida personal y social, ya que pueden realizar el intercambio en su casa, ya sea durante toda la noche o por el día en varias fases. A pesar de ello, la duración del tratamiento en nuestra muestra no obtuvo impacto estadísticamente significativo sobre la calidad de vida. Por el contrario, el tiempo que llevan en tratamiento dialítico sí se asoció significativamente con afectación de ciertos dominios de la calidad de vida, lo cual no resulta extraño debido a algunos factores precipitantes que van en aumento como la edad y las comorbilidades en estos pacientes, comentado igualmente en la revisión de Andreu donde asevera que a pesar de los grandes avances técnicos en los tratamientos, la supervivencia no ha experimentado grandes mejoras ${ }^{29}$.

Respecto a la posible relación entre los dominios y los parámetros analíticos, en nuestra muestra se ha hallado cierta asociación de algunos de los parámetros como el $\mathrm{Kt} / \mathrm{V}$ con los dominios rol físico $(p=0,023)$, dolor corporal $(p=0,036)$ y salud general $(p=0,018)$. Igualmente parece que en nuestra muestra existe relación entre parámetros como el potasio, la hemoglobina, la urea, el calcio y el fósforo, con diferentes dominios que caracterizan la calidad de vida percibida por el paciente. Dado que dichos parámetros analíticos dependen directamente de la dosis de diálisis administrada, parece que el
$\mathrm{Kt} / \mathrm{V}$ podría ser un indicador a tener en cuenta a la hora de valorar la calidad de vida percibida por el paciente, en concordancia con la actualización de las guías de práctica clínica KDOQI, en las cuales se sugieren diálisis más frecuentes y/o de mayor duración ${ }^{30}$.

Igualmente la revisión bibliográfica de Sierra concluye que un adecuado ajuste en la dosis de diálisis contribuye a una mejora de la calidad de vida de los pacientes ${ }^{31}$.

Por otro lado, el grupo de Guerra observó en una muestra de 354 pacientes, relación de la calidad de vida con algunas variables de laboratorio como la albúmina, creatinina, ferritina y nitrógeno ureico ${ }^{32}$. Se debe mencionar que este estudio se llevó a cabo únicamente sobre pacientes en hemodiálisis, que obtuvieron menores puntuaciones en los dominios de la calidad de vida y en la dosis de diálisis ( $\mathrm{K} / \mathrm{V}$ de 1,46 vs. 1,64 de nuestra muestra). Los parámetros analíticos en conjunto pueden relacionarse con el estado nutricional de los pacientes, variable que afecta directamente a la calidad de vida, mejorando la tolerancia al tratamiento y disminuyendo la comorbilidad asociada ${ }^{33}$.

La limitación del tamaño muestral debe ser tenida en cuenta, a pesar de ser esta muestra de pacientes quienes cumplían los criterios de selección.

Se puede decir que a partir de los resultados obtenidos en nuestra muestra, observamos que el tratamiento renal sustitutivo, ya sea HD o DP posee un gran impacto a todos los niveles en los pacientes, obteniendo una puntuación global de $64,6 \%$ en cuanto a la satisfacción de su percepción de la calidad de vida. Respecto a la técnica de tratamiento, la DP obtiene mejores puntuaciones en determinados dominios de la calidad de vida, al igual que el catéter peritoneal como acceso para diálisis. También parecen obtener mejores puntuaciones en algunos dominios los que menos tiempo llevan en diálisis y los que mejores parámetros analíticos presentan, especialmente en el Kt/V.

\section{Agradecimientos}

Queremos agradecer la participación a los pacientes de la Unidad de Diálisis del Hospital Universitario Fundación Jiménez Díaz, por su paciencia y dedicación para ayudarnos a llevar a cabo este proyecto. 
Recibido: 05-11-18

Revisa: 04-01-19

Modificado: 25-02-19

Aceptado: 05-03-19

\section{Bibliografía}

1. Informe de diálisis y trasplantes 2016. [Internet]. Sociedad Española de Nefrología. 2017. [Consultado 15 dic 2017]. Disponible en: http://www.ont.es/ infesp/Registros/InformeREER_2016.pdf

2. Cuevas-Budhart MA, Saucedo RP, Romero G, García JA, Hernández A. Relación entre las complicaciones y la calidad de vida del paciente en hemodiálisis. Enferm Nefrol 2017;20(2):112-9.

3. Ahís $P$, Peris IC, Meneu $M$, Pérez CM, Bonilla $B$, Panizo N. Impacto subjetivo de las estrategias no farmacológicas de mejora de la calidad de vida y el cumplimiento terapéutico en pacientes en hemodiálisis. Enferm Nefrol 2017;20(1):22-7.

4. Hernández S, García D, Santos A, González G, Brazález M, Garatachea N. Valoración física, condición física y calidad de vida en pacientes con diferentes tratamientos renales sustitutivos. Enferm Nefrol 2015;18(2):81-8.

5. Zazzeroni L, Pasquinelli G, Nanni E, Cremonini V. Comparison of Quality of Life in Patients Undergoing Hemodialysis and Peritoneal Dialysis: a Systematic Review and Meta- Analysis. Kidney Blood Press Res 2017;42:717-27.

6. Barbero E, Tejeda E, Herrera C, Monserrat S, Gascó $\mathrm{N}$, Junyent $\mathrm{E}$. Estudio comparativo del estado físico, mental y percepción de calidad de vida relacionada con la salud de los pacientes en diálisis. Enferm Nefrol 2016;19(1):29-35.

7. Pérez C, Riquelme G, Scharager J, Armijo I. Relación entre calidad de vida y representación de enfermedad en personas con enfermedad renal crónica terminal en tratamiento con hemodiálisis. Enferm Nefrol 2015;18(2):89-96.
8. Queeley GL, Campbell ES. Comparing treatment modalities for end-stage renal disease: a meta-analysis. Am Health Drug Benefits 2018;11(3): 118-27.

9. Dąbrowska-Bender M, Dykowska G, Žuk W, Milewska $M$, Staniszewska $A$. The impact on quality of life of dialysis patients with renal insufficiency. Patient Prefer Adherence 2018;12:577-83.

10. Chen JY, Wan EYF, Choi EPH, Chan AKC, Chan KHY, Tsang JPY, et al. The Health-Related Quality of Life of Chinese Patients on Hemodialysis and Peritoneal Dialysis. Patient 2017;10(6):799-808.

11. Kang SH, Do JY, Lee SY, Kim JC. Effect of dialysis modality on frailty phenotype, disability, and health-related quality of life in maintenance dialysis patients. PLoS One 2017;12(5):e0176814.

12. Grupo Español Multidisciplinar del Acceso Vascular (GEMAV). Guía Clínica Española del Acceso Vascular para Hemodiálisis. Enferm Nefrol 2018;21 (Supl 1):S1-256.

13. Tattersall J, Farrington K, Gentile G, Kooman J, Macias Núñez JF, Nistor I, et al; European Renal Best Practice Guidelines Group and the European Union of Geriatric Medicine Societies (EUGMS) collaborative group for the production of guidelines on the management of older patients with CKD. Is Kt/V useful in elderly dialysis patients? Pro and Con arguments. Nephrol Dial Transplant 2018;33(5):742-50.

14. Leyva E, Argudín R, Mora SR, Capote L, Rupalé I, Moret $Y$. Evaluación de la calidad de vida relacionada con salud en pacientes en hemodiálisis periódica utilizando el KDQOL - SFTM. MediSur 2015;13 (4):508-16.

15. Peipert JD, Bentler PM, Klicko K, Hays RD. Psychometric Properties of the Kidney Disease Quality of Life 36-Item Short-Form Survey (KDQ0L-36) in the United States. Am J Kidney Dis 2018;71(4): 461-8.

16. Adell M, Casadó L, Andújar J, Solá E, Martínez E, Salvadó T. Valoración de la calidad de vida relacionada con la salud en la enfermedad renal crónica terminal mediante un cuestionario de resultados percibidos por los pacientes. Enferm Nefrol 2016; 19(4):331-40. 
17. Adell M, Salvadó T, Casadó L, Andújar J, Ramiro M, Mencía A. Valoración de la calidad de vida y técnicas de diálisis mediante un cuestionario de resultados percibidos por el paciente. Enferm Nefrol 2015; 18 Suppl(1):S101-37.

18. Martín F, Reig A, Sarró F, Ferrer R, Arenas D, González F, Gil T. Evaluación de la calidad de vida en pacientes de una unidad de hemodiálisis con el cuestionario Kidney Disease Quality of Life - Short Form (KDQOL-SF). DyT 2004;25(2):79-92.

19. Arroyo E, Arana AI, Garrido R, Crespo R. Análisis de la sobrecarga del cuidador del paciente en diálisis. Enferm Nefrol 2018;21(3):213-24.

20. Barros L, Herazo Y, Aroca G. Calidad de vida relacionada con la salud en pacientes con enfermedad renal crónica. Rev Fac Med 2015;63(4):641-7.

21. Costa G, Pinheiro MB, Medeiros SM, Costa RR, Cossi M. Calidad de vida en pacientes con insuficiencia renal crónica en hemodiálisis. Enferm Global 2016;43(1):59-74.

22. Ho YF, Li IC. The influence of different dialysis modalities on the quality of life of patients with end-stage renaldisease: A systematic literature review. Psychol Health 2016;31(12):1435-65.

23. Homaie E, Mostafavi H, Delavari S, Mostafavi S. Health-related Quality of Life in Patients on Hemodialysis and Peritoneal Dialysis: a Meta-Analysis of Iranian Studies. Iran J Kidney Dis 2015;9(5):386-93.

24. Wakeel J, Harbi A, Bayoumi M, Suwaida K, Ghonaim $M$, Mishkiry A. Quality of life in hemodialysis and peritoneal dialysis patients in Saudi Arabia. Ann Saudi Med 2012;32(6):570-4.

25. Atapour A, Nasr S, Momeni Boroujeni A, Taheri D, Dolatkhah S. A comparison of the quality of life of the patients undergoing hemodialysis versus peritoneal dialysis and its correlation to the quality of dialysis. Saudi J Kidney Dis and Transpl 2016;27 (2):270-80.

26. Gonçalves FA, Dalosso IF, Borba JM, Bucaneve J, Valerio NM, Okamoto CT, et al. Quality of life in chronic renal patients on hemodialysis or peritoneal dialysis: a comparative study in a referral service of Curitiba - PR. J Bras Nefrol 2015;37(4):467-74.

27. Ho Y, Li I. The influence of different dialysis modalities on the quality of life of patients with end-stage renal disease: A systematic literature review. Psychol Health 2016;31(12):1435-65.

28. Iyasere $0 U$, Brown EA, Johansson $L$, Huson $L$, Smee $J$, Maxwell AP. Quality of Life and physical function in older patients on dialysis: a comparison of assisted peritoneal dialysis with hemodialysis. Clin J Am Soc Nephrol 2016;11(3):423-30.

29. Andreu D, Hidalgo MA, Moreno MC. La supervivencia de las personas sometidas a diálisis. Enferm Nefrol 2013;16(4):278-80.

30. National Kidney Foundation. KDOQI clinical practice guideline for hemodialysis adequacy: 2015 update. Am J Kidney Dis 2015;66(5):884-930.

31. Sierra R, Medina L, Ciriza A. Revisión bibliográfica sobre dos modelos de medición del Kt/V: OCM vs fórmula de Daurgidas. Comunicaciones presentadas al XXXIV Congreso Nacional Seden; Pamplona 2010;315-9.

32. Guerra V, Sanhueza 0, Cáceres M. Calidad de vida de personas en hemodiálisis crónica: relación con variables sociodemográficas, médico-clínicas y de laboratorio. Rev Latino-Am Enfermagem 2012;20 (5):1-10.

33. Pereira MC, Queija L, Blanco A, Rivera IA, Martínez VE, Prada Z. Valoración del estado nutricional y consume alimentario de los pacientes en terapia renal sustitutiva mediante hemodiálisis. Enferm $\mathrm{Ne}$ frol 2015;18(2):103-11.

Este artículo se distribuye bajo una Licencia Creative Commons Atribución-NoComercial 4.0 Internacional. https://creativecommons.org/licenses/by-nc/4.0/

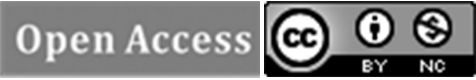

